\title{
Enantioselective Addition of Diethylzinc to Aldehydes with Easily Prepared Chiral Thiazolidine Catalysts
}

\author{
Myung-Jong Jin * and Sang-Han Kim \\ School of Chemical Science \& Engineering, Inha Liniversity, Inchon t02-751, Korea \\ Received October 31, 2001
}

Keywords : Enantioselective addition. Diethỵlzinc. Aldehỵde. L-Cysteine ester. Chiral thiazolidine.

Asymmetric metal cataly sis is now recognized as the most promising area in the synthesis of optically active organic compounds. One attractive method that leads to the formation of optically active secondary alcohols is catalytic enantioselective addition of dialkytzinc reagent to aldehỵdes. ${ }^{1}$ Numerous elegant and efficient cataly sts have been developed for this purpose. in which most of them are based on amino alcohols. ${ }^{2}$ diols. ${ }^{3}$ diamines. ${ }^{+}$and their derivatives. ${ }^{5}$ In spite of the variety of chiral catalysts. the develoment of catalysts obtainable by simple synthetic routes remains still as an active research subject. We have easily prepared 4carboethoxy-1.3-thiazolidines 2-5 from L-cysteine ester through one step. Previously, thiazolidine 4 was found to catalyze the dialkylzinc-aldehyde addition. ${ }^{\circ}$ Following on from previous work we wish to report the results on the enantioselective dialkylzinc-aldehyde addition in the presence of several N.S-chelate thiazolidine ligands.
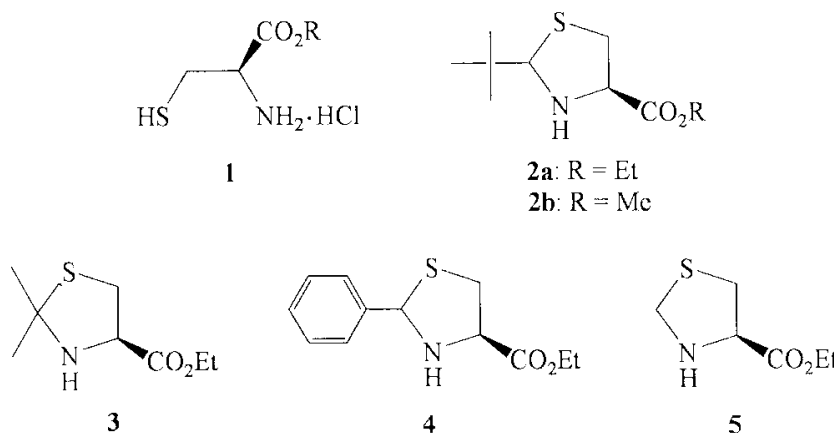

Chiral thiazolidines 2-5 were easily prepared by condensation of L-cysteine ester 1 with the corresponding carbonyl conpounds. Thiazolidines 2 and + were obtained as inseparable $c a$. 1:1.9 diastereomeric mixtures. ${ }^{7}$ (2R.4R)-cis-Thiazolidines were assigned to be major diastereomers on a combination of the ${ }^{1} \mathrm{H}$ NMR data and the previous studies. ${ }^{8}$ The catalytic behavior of the ligands $\mathbf{2 - 5}$ for the diethylzincaldehyde addition was then examined. The reaction conditions and results are sumntarized in Table 1 . All the aldehydes were converted to the corresponding (S)-alcohols with moderate to excellent enantioneric excess in high yields. Enantioselectivity was somewhat dependent on the amount of the ligand. Increasing the amount of ligand from 4 to $8 \%$ led to a small increase in the enantioselectivity (entries 1-3) and $6 \mathrm{~mol} \%$ of ligand was enough to afford good enantioselectivity and reactivity. It is noteworthy that the ee
Table 1. Enantioselective Addition of Diethylzinc to Aldehydes"

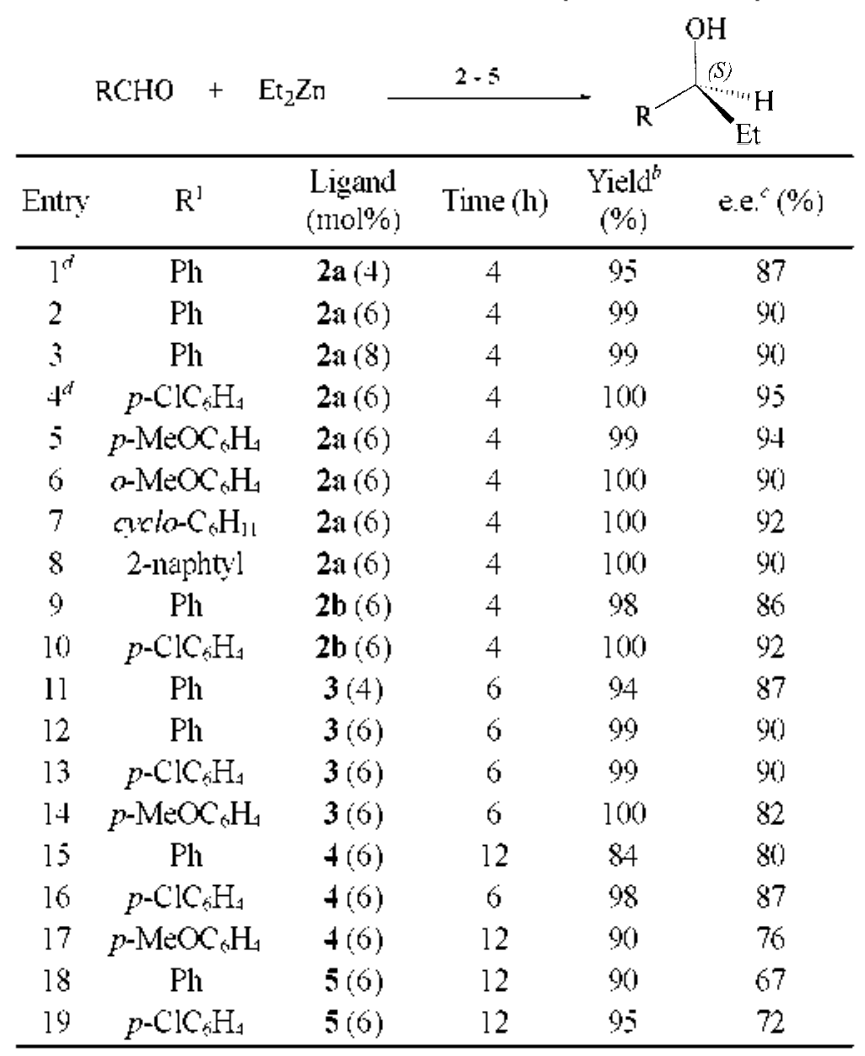

Reactions were carried out in hexane at 0 " $\mathrm{C} \rightarrow$ RT. Molar ratio, Et $2, n$ : aldelyyde : ligand $=2: 1: 0.04-0.08$. Absolute contiguration was assigned by the sign of the optical rotation and elution order from a chiral column. "Measured as "o-conversion into the product by $\mathrm{GC}$. "determined by HPLC analysis (chiralcel OD column) or GC analysis $(\beta-$ DEX chiral columns.

of the product depends on the size of substituents on thiazolidine ring. Thiazolidine $\mathbf{2 a}$ having larger carboalkoxy moiety showed higher enantioselectivity than $\mathbf{2 b}$ (entries 2 . 4 versus 9, 10). Although $R$ group affects the enantioselectivity. the bulkiness of C2-substituent on the thiazolidine ring is more important. Higher enantioselectivity was obtained with increasing size of the C2-substituent. Introduction of tent-butyl group on $\mathrm{C} 2$ led to the most improved enantioselectivity in the range of $90-95 \%$ ee. It is interesting that high enantioselectivity could be obtained with diastereomeric mixture 2. This result suggests that the chirality of substituent on $\mathrm{C} 2$ is less important role for the stereochemical outcome. Each of diasteromers that differ in the 
configuration at $\mathrm{C} 2$ would generate similar asymmetric induction environment. Accordingly. diastereomerically pure ligand is not necessarily required in order to obtain high enantioselectivity.

In conclusion. we have prepared chiral thiazolidines 2-5 derived from L-cysteine ethyl ester. In particular $2 \mathbf{a}$ could catalyze efficiently the enantioselective addition of dialkylzinc to aldehydes.

\section{Experimental Section}

Reactions were carried out under an inert nitrogen atmosphere using dried glassware. All the commercially available reagents were used without further purification. NMR spectra were recorded on a Bruker AC 250 NMR spectrometer. Optical rotation were measured with a Perkin-Elmer 241 polarimeter. IR spectra were obtained on a Bruker IFS-48.

Preparation of 2-substituted-1,3-thiazolidines-4-carboxylate $2-5$. Thiazolidines $2 \mathrm{a}^{6.8}$ and $t^{6}$ have been previously reported. According to the previous method, 2-5 were prepared by the reaction of L-cysteine ester hydrochloride with the corresponding carbonyl conpounds.

2-tert-Butyl-t-carboethoxy-1,3-thiazolidine (2a). Yield 93\%: colorless liquid: $[\alpha]_{\mathrm{D}^{3 i}}-153.2\left(\mathrm{c}=1.0 . \mathrm{CHCl}_{3}\right)$; major diastereomer: ${ }^{\mathrm{l}} \mathrm{H}-\mathrm{NMR}\left(\mathrm{CDCl}_{3}, 250 \mathrm{MHz}\right) \delta 4.40$ (s. IH. $\mathrm{SC} H \mathrm{~N}$ ). 4.18 (q. $J=7.3 \mathrm{~Hz} .2 \mathrm{H} . \mathrm{OCH}_{2} \mathrm{CH}_{3}$ ), 3.72 (dd. $J=$ 8.3. $\left.6.8 \mathrm{~Hz} . \mathrm{lH}, \mathrm{SCH}_{2} \mathrm{CH}\right), 3.23(\mathrm{dd}, J=8.4 .6 .8 \mathrm{~Hz} . \mathrm{lH}$. $\mathrm{SCH} H_{2} \mathrm{CH}$ ), 2.62 (t. $J=J^{\prime}=10.0 \mathrm{~Hz}$. IH. SCH$H_{-} \mathrm{CH}$ ). 2.17 (br. $1 \mathrm{H}, \mathrm{NH}), 1.24\left(\mathrm{t}, J=7.2 \mathrm{~Hz}, 3 \mathrm{H} . \mathrm{OCH}_{2} \mathrm{CH}_{3}\right), 1.02(\mathrm{~s}, 9 \mathrm{H}$. $\left.\mathrm{C}\left(\mathrm{CH}_{3}\right)_{3}\right)$; minor diasteromer: $4.40(\mathrm{~s}, 1 \mathrm{H} . \mathrm{SCHN}) .4 .15(\mathrm{q} . J$ $=5.3 \mathrm{~Hz}, 2 \mathrm{H} . \mathrm{OCH}_{2} \mathrm{CH}_{3}$ ), 4.04 (t. $J=J^{\prime}=6.0 \mathrm{~Hz} .1 \mathrm{H}$. $\mathrm{SCH}_{2} \mathrm{CH}$ ), 3.07 (dd. $J=8.6,6.4 \mathrm{~Hz} . \mathrm{IH} . \mathrm{SCH}_{2} \mathrm{CH}$ ), 2.94 (dd, $J=8.2 .6 .0 \mathrm{~Hz}, 1 \mathrm{H}, \mathrm{SC} H_{2} \mathrm{CH}$ ), 2.17 (br. $\mathrm{lH}$. NH) 1.23 (t. $\left.J=7.2 \mathrm{~Hz} .3 \mathrm{H} . \mathrm{OCH}_{2} \mathrm{CH}_{3}\right), 0.92$ (s. $\left.9 \mathrm{H}, \mathrm{C}\left(\mathrm{CH}_{3}\right)_{3}\right)$ : IR (neat) $v=01736 \mathrm{~cm}^{-1}$ : MS (EI) $m z 217\left(\mathrm{M}^{-}\right)$.

2,2-Dimethyl-4-carboethoxy-1,3-thiazolidine (3). yield $81 \%$ : colorless liquid, $[\alpha]_{\mathrm{D}}{ }^{301}-178.6\left(\mathrm{c}=1.4, \mathrm{CHCl}_{3}\right) .{ }^{1} \mathrm{H}$ NMR $\left(\mathrm{CDCl}_{3 .} 250 \mathrm{MHz}\right) \delta 4.18$ (q. $J=7.2 \mathrm{~Hz}, 2 \mathrm{H}$. $\left.\mathrm{OC}_{3} \mathrm{CH}_{3}\right) .4 .02\left(\mathrm{dd}, J=9.1,7.0 \mathrm{~Hz}, 1 \mathrm{H} . \mathrm{SCH}_{2} \mathrm{CH}\right), 3.38$ (dd $J=10.5 .7 .0 \mathrm{~Hz} .1 \mathrm{H}, \mathrm{SCH} \mathrm{C}_{2} \mathrm{CH}$ ). 2.95 (br, $\mathrm{lH}$. NH). 2.96 (dd. $J=10.3 .9 .2 \mathrm{~Hz} .1 \mathrm{H}$. $\mathrm{SCH} \mathrm{H}_{2} \mathrm{CH}$ ). 1.65 (s. $3 \mathrm{H} . \mathrm{CCH}_{3}$ ). 1.47 (s. $3 \mathrm{H} . \mathrm{CCH}_{3}$ ). 1.24 (t. $J=7.1 \mathrm{~Hz} .3 \mathrm{H} . \mathrm{OCH}_{2} \mathrm{CH}_{3}$ ): IR (neat) $v_{c}=01742 \mathrm{~cm}^{-1}$ : MS (EI) $m z 189\left(\mathrm{M}^{-}\right)$.

4-Carboethoxy-1,3-thiazolidines (5). yield 67\%; colorless liquid, $[\alpha]_{\mathrm{D}}{ }^{2(1)}-145.4\left(\mathrm{c}=1.1 . \mathrm{CHCl}_{3}\right)$. ${ }^{1} \mathrm{H}-\mathrm{NMR}\left(\mathrm{CDCl}_{3}\right.$. $250 \mathrm{MHz}) \delta 4.35\left(\mathrm{~d} . J=9.6 \mathrm{~Hz}, 1 \mathrm{H}, \mathrm{SC} H_{2} \mathrm{~N}\right), 4.20$ (q. $J=$ $\left.7.5 \mathrm{~Hz} .2 \mathrm{H} . \mathrm{OCH}_{2} \mathrm{CH}_{3}\right), 4.08\left(\right.$ d. $\left.J=9.6 . \mathrm{Hz} .1 \mathrm{H} . \mathrm{SCH} \mathrm{H}_{2} \mathrm{~N}\right)$. $3.79\left(\mathrm{t} . J=J^{\prime}=7.5 \mathrm{~Hz}, \mathrm{lH} . \mathrm{SCH}+\mathrm{CH}\right) .3 .22(\mathrm{dd} . J=10.0,4.5$ Hz. $1 \mathrm{H}$. SCH $H_{2} \mathrm{CH}$ ), 2.82 (dd. $J=13.8,8.0 \mathrm{~Hz} .1 \mathrm{H}, \mathrm{SC} H_{2} \mathrm{CH}$ ), 2.32 (br, $\mathrm{lH} . \mathrm{N} H), 1.26\left(\mathrm{t}, J=7.1 \mathrm{~Hz}, 3 \mathrm{H} . \mathrm{OCH}_{2} \mathrm{CH}_{3}\right.$ ); IR (neat) $v_{c}=01736 \mathrm{~cm}^{-1}$ : MS (EI) $m z 161\left(\mathrm{M}^{-}\right)$.
General Procedure for the enantioselective addition of diethylzinc to aromatic aldehydes. To a solution of thiazolidine $(0.06 \mathrm{mmol})$ in hexane $(2.0 \mathrm{~mL})$ was added $\mathrm{Et} Z \mathrm{Zn}(2.0$ $\mathrm{mL}, 1.0 \mathrm{M}$ in hexane) at $0^{\circ} \mathrm{C}$. The mixture was allowed to warm to RT. Aldehyde $(1.0 \mathrm{mmol})$ was added dropwise and the reaction mixture was stirred at RT for a given time, observing the progress of the reaction by $\mathrm{GC}$. The reaction was quenched at $0{ }^{\circ} \mathrm{C}$ by addition of $1.0 \mathrm{M} \mathrm{HCl}$ and the resulting mixture was extracted with $\mathrm{CH}_{2} \mathrm{Cl}_{2}$. After removal of solvent the residue was purified by flash cluromatograply on silica gel (hexane $/ \mathrm{AcOEt}=8 / 2$ ). The enantiomeric excess was determined by HPLC (chiralcel OD column) or GC ( $\beta$ DEX chiral columin)

Acknowledgment. This work was supported by the Center for Advanced Bioseparation Teclunology. Inha University

\section{References}

1. For reviews: (a) Noyori, R:; Kitamura, M. Angew: Chem. Int. Ed. Engl. 1991. 30.49. (b) Soai. K.: Niwa. S. Chent Rev 1992. 92. 833.

2. (a) Sola. L.: Reddy. K. S.: Vidal-Ferran. A.: Moyano. A.: Perica. M. A; Riera, A.; Alvarez-Larena. A.; Piniella. J. J. Org. Chem. 1998. 63, 7078. (b) Cho, B. T.: Chun, Y. S. Tetrahedron Asmmeto 1998. 9. 1489. (c) Tanner. D.: Korno, T: Guijarro. D.: Andersson. P. G. Tetrahedron 1998. 5t. 14213. (d) Bolm. C.: Muniz. K. Hildebrand. .T. P. Org. Lett. 1999. 1. 491. (e) Xu. Q.: Pan1. X: Chan1. A.: Yang. T. Tetrahedron Lett. 2001. \$2.6171.

3. (a) Rosini, C.; Franzini. L.; Pini, D; Salvadori, P. Terohedron Asymmetry 1990. 1. 587. (b) Schmit, B.; Seebach. D. Angew: Chem. Iht Ed. Engl 1991, 30,1321 . (c) Weber. B.; Seebach. D. Tetwatedron 1994. 50.7473. (d) Huang. W.S.: Hu. Q.-S.: Pu. L. J. Org. Chen 1999.64.7940

4. (a) Soai. K.: Niwa. S.: Yamada. Y.: Inoue. H. Tetrahedon Lett 1987. 28, 4841. (b) Rosini, C.; Franzini, L.: Iuliano, A.: Pini. D. Salvadori. P. Tetrahedron Asummetry 1991, 2, 363. (c) Conti. S. Falorni, M.: Giacomelli, G.: Soccolini. F. Tetohledron 1992, 48. 8993 .

5. (a) Yoshioka. M.: Kawakita. T.: Ohno. M. Tetrohedron Lett. 1989. 30. 1657. (b) Katsuji. I.: Kimula. Y.: Okamura. H.: Katsuki. T. Synlett 1992. 573. (c) Kang. J.: Kim. J. W. Lee. J. W.: Kim. D. S.: Kim. J. L. Bull. Konam Chem. Soc. 1996, 17, 1135. (d) Vettel. S: Lutz. C: Diefenbach. A.: Harderlein. G.: Hammerschmidt. S.: Kuhling. K.: Mofid. M.-R.: Zimmermann. T.: Knochel. P. Tetrohedrot: Astmmenv 1997. 8. 779. (e) Ramon1. D. T.: Yus. M. Tetrahedront Astmmenv 1997. 8. 2479. (d) Tinl. M.-T.: Kiml. S.-H.: Jung. J.A. Lee, H.-Y. Bull. Korean Chem. Soc. 2000, 21, 33 .

6. Kim, S.-H.: Ahn, S.-J; Chung, S.-T; Jin, M.-J. d. Ind \& Eng. Chem. 1997, 3, 37

7. The diastereomeric ratio was be deternined by the intensity ratio of two singlets for $\mathrm{C} 2$ proton in the ${ }^{\mathrm{H}} \mathrm{H}$ NMR spectrum which is attributed to two diastereomers.

8. Calmes. M.: Escale, F.: Paolini, F. Tetrahedron: Asymmeny 1997. 8.3691

9. Brunner. H.: Becker, R:- Riepl. G. Onganometallics 1984. 3. 1354 